\title{
On Extension the Stability Region of Implicit-Explicit Linear Multistep Methods for Ordinary Differential Equation
}

\author{
Ghanim M. S. Abdullah \\ Department of Mathematic / College of Education \\ University of Mosul
}

Received

14 / 03 / 2011
Accepted

15 / 09 / 2011

\begin{abstract}
الملخص
إن دراسـة خاصية استقرارية طرق متعددة الخطوات (الصريحة - الضمنية) لمعادلات التفاضلية الاعنياديـة تم تحليلها على مناطق الاستقرارية المعرفة من خلال المسائل الاختياريـة وان هذه الدراسـة أجريت على الطرق متعددة الخطوات الاعتيادية بالنسبة للمعادلات التفاضلية

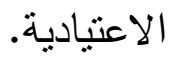

في هـذا البحـث تـم إعطـاء فكـرتين أساسـيتين لتوسـيع منــاطق الاسـتقرارية للطـرق

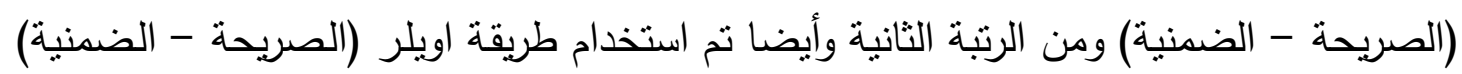

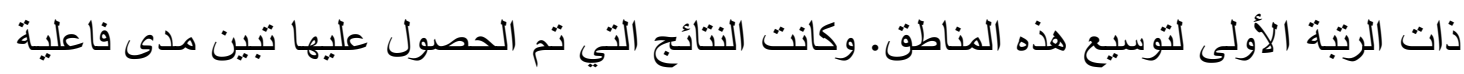
استخدام الطرق المعدلة عن مثنلاتها.
\end{abstract}

\begin{abstract}
Stability properties of implicit - explicit (IMEX) of linear multistep methods for ordinary differential equations are analyzed on the basis of stability regions defined by using scalar test equations. This study is closely related to the stability analysis of the standard linear multistep methods for ordinary differential equation.

In this paper we give two idea to extend the stability region for the second - order IMEX method, also have extended the region IMEX Euler method, the simplest IMEX method of first order, numerical results are presented which show superiority of improving method.
\end{abstract}

\section{1- Introduction}

In this paper we look at the general ODE problem

$$
\frac{d u}{d t}=f(t, u(t))+g(t, u(t))
$$


Where $\boldsymbol{f}$ represent the stiff part and $\boldsymbol{g}$ represents the non stiff part. In many applications, $\boldsymbol{f}$ is linear and $\boldsymbol{g}$ is non linear. In order to solve such equation efficiently, a special type of numerical method called implicitexplicit (IMEX) method is often used, which is obtained by applying an implicit formula with a good stability property to the $\boldsymbol{f}$ term and an explicit formula to the $\boldsymbol{g}$ term $[7,10]$.

One of the simplest examples is the IMEX Euler method:

$u_{n+1}=u_{n}+\Delta t f\left(t_{n+1}, u_{n+1}\right)+\Delta t g\left(t_{n}, u_{n}\right)$

Which is obtained by applying the implicit Euler formula to the $f$ term and explicit Euler formula to the $\boldsymbol{g}$ term.

Here, $\Delta \boldsymbol{t}$ is the step-size, $\boldsymbol{t}_{\boldsymbol{n}}=\boldsymbol{t}_{\mathbf{0}}+\boldsymbol{n} \boldsymbol{\Delta t}$, and $\boldsymbol{u}_{\boldsymbol{n}}$ denotes an approximate value of $\boldsymbol{u}\left(\boldsymbol{t}_{n}\right)$.

This method is of order one in accuracy.

In this paper, we discuss IMEX methods of linear multi-step type.

For numerical solution of (1.1) we consider IMEX linear multi-step

method $\sum_{j=0}^{k} \alpha_{j} u_{n+j}=\Delta t \sum_{j=0}^{k} \beta_{j} f\left(t_{n+j}, u_{n+j}\right)+\Delta t \sum_{j=0}^{k-1} \beta_{j}^{*} g\left(t_{n+j} u_{n+j}\right)$

Here, $\boldsymbol{\alpha}_{j}, \boldsymbol{\beta}_{j}$ denote coefficients of $\mathrm{k}$-step linear multi-step method. Coefficients $\boldsymbol{\beta}_{j}^{*}$ are determined by $\boldsymbol{\beta}_{j}^{*}=\boldsymbol{\beta}_{j}+\boldsymbol{\beta}_{k} \gamma_{j}$ with coefficients $\gamma_{j}$ of a suitable extrapolation to incorporate $\beta_{\boldsymbol{k}}$ into the other coefficients [10].

For the study of stability of such IMEX methods, Frank et al.[ 5] proposed

$\frac{d u}{d t}=\lambda u(t)+\mu u(t), \lambda, \mu \in C$

As a test equation (see $\operatorname{Refs}[1,2,5,7]$ for related studies), where $\lambda \boldsymbol{u}(\boldsymbol{t})$ and $\boldsymbol{\mu} \boldsymbol{u}(\boldsymbol{t})$ correspond to $\boldsymbol{f}$ term and $\boldsymbol{g}$ term in (1.1).respectively.

Application of method (1.3) to this test equation yields the difference equation

$\sum_{j=0}^{k} \alpha_{j} u_{n+j}=z \sum_{j=0}^{k} \beta_{j} u_{n+j}+\omega \sum_{j=0}^{k-1} \beta_{j}^{*} u_{n+j}, z=\Delta t \lambda, \omega=\Delta t \mu$

The stability region $S$ of the IMEX method is defined as a region of the parameters $(z, \omega)=(\Delta t \lambda, \Delta t \mu)$ such that the zero solution of (1.5) is asymptotically stable. we can study the stability of the method on the basis of $\boldsymbol{S}$ in the similar manner as in the case of the standard linear multi-step methods $[8,9]$.

However, $\boldsymbol{S}$ is a region in $\phi^{2}$ it is not easy to construct a new scheme by adjusting the parameters $\boldsymbol{\alpha}_{j}, \boldsymbol{\beta}_{j}$ and $\gamma_{j}$ so as to enlarge the stability region $\boldsymbol{S}$.

To overcome this difficulty, we consider, in analogy to (1.4), 
the test equation

$$
\frac{d u}{d t}=\lambda u(t)+\mu u(t-\tau), \lambda, \mu \in C
$$

originally proposed by Barwell in 1975 [10] for the study of the stability of numerical method for delay differential equation $\left(\mathrm{DDE}_{\mathrm{s}}\right)$ with constant delay. Here, $\tau>\mathbf{0}$ is a constant delay. when the step-size $\Delta \boldsymbol{t}$ is given in the form $\Delta t=\frac{\tau}{m}, \quad(\mathrm{~m} \geq 1 ;$ integer $)$,

an IMEX method can be applied to $\mathrm{DDE}_{\mathrm{s}}$ of form [2]

$$
\frac{d u}{d t}=f(t, u(t))+g(t, u(t-\tau))
$$

\section{2- Stability regions}

We assume that the linear multi-step method determined from $\alpha_{j}, \beta_{j}$ is of order $\boldsymbol{p} \geq \mathbf{1}$, and $\gamma_{j}$ satisfy for any sufficiently smooth function $\varphi(t)[10]$ and $\sum_{j=0}^{k-1} j^{q} \gamma_{j}=k^{q}, \mathrm{q}=0,1, \ldots, \mathrm{p}-1$

For example in the case $\boldsymbol{k}=\boldsymbol{p}=\mathbf{2}$, from the condition $\gamma_{0}+\gamma_{1}=\mathbf{1}, \gamma_{1}=\mathbf{2}$ coefficients $\gamma_{1}, \gamma_{0}$ are uniquely determined as $\gamma_{1}=\mathbf{2}, \gamma_{0}=-\mathbf{1}$ which gives a linear extrapolation determined from (2.1) and satisfies [10] $\xi^{k}-(\xi-1)^{k}-\sum_{j=0}^{k-1} \gamma_{j} \xi^{j}$

The pair gives a polynomial extrapolation. Condition (2.1) assures that the local error of method (1.3) is $\boldsymbol{O}\left(\Delta t^{p+1}\right)$.

Here, we assume that $\boldsymbol{u}\left(\boldsymbol{t}_{n}+\Delta t\right) \approx \boldsymbol{u}\left(\boldsymbol{t}_{n}\right)$, as $\Delta t \rightarrow \mathbf{0}$.

So, we can say that $\delta u\left(t_{n}+\Delta t\right) \approx \delta u\left(t_{n}\right), 0 \leq \delta \leq 1, a s \Delta t \rightarrow 0$

In this paper we shall discuss two cases:

- First:suppose that $\Phi=\delta \boldsymbol{u}_{n+k}+\delta \boldsymbol{u}_{n+k-1}$

Then, the IMEX linear multi-step method (1.3) for equation (2.2) is represented as

$$
\Phi+\sum_{j=0}^{k} \alpha_{j} u_{n+j}=\Delta t \sum_{j=0}^{k} \beta_{j} f\left(t_{n+j}, u_{n+j}\right)+\Delta t \sum_{j=0}^{k-1} \beta_{j}^{*} g\left(t_{n+j}, u_{n+j}\right)
$$

Or

$$
(1+\delta)\left(\alpha_{k} u_{n+k}+\alpha_{k-1} u_{n+k-1}\right)+\sum_{j=0}^{k-2} \alpha_{j} u_{n+j}=\Delta \sum_{j=0}^{k} \beta_{j} f\left(t_{n+j}, u_{n+j}\right)+\Delta \sum_{j=0}^{k-1} \beta_{j}^{*} g\left(t_{n+j}, u_{n+j}\right)
$$

- Second: if we assume that $\Phi=\delta \boldsymbol{u}_{n+k}+\delta \boldsymbol{u}_{n+k-1}+\ldots+\delta \boldsymbol{u}_{n}$ This idea we can not applied except when we have a method such that $\sum_{j=0}^{k} \alpha_{j} u_{n+k}, k>2$. 
So, the IMEX linear multi-step method(1.3) for equation(2.2) is represented as

$\Phi+\sum_{j=0}^{k} \alpha_{j} u_{n+j}=\Delta t \sum_{j=0}^{k} \beta_{j} f\left(t_{n+j}, u_{n+j}\right)+\Delta t \sum_{j=0}^{k-1} \beta_{j}^{*} g\left(t_{n+j}, u_{n+j}\right)$,

Or

$(1+\delta) \sum_{j=0}^{k} \alpha_{j} u_{n+j}=\Delta v \sum_{j=0}^{k} \beta_{j} f\left(t_{n+j}, u_{n+j}\right)+\Delta v \sum_{j=0}^{k-1} \beta_{j}^{*} g\left(t_{n+j}, u_{n+j}\right)$

where $\boldsymbol{\alpha}_{j}, \boldsymbol{\beta}_{j}$ denote coefficient of a k-step linear multi-step method.

Application of method (2.3) and (2.4) to the test equation yields the difference equations

$\Phi+\sum_{j=0}^{k} \alpha_{j} u_{n+j}=z \sum_{j=0}^{k} \beta_{j} u_{n+j}+\omega \sum_{j=0}^{k-1} \beta_{j}^{*} u_{n+j}$,
$z=\Delta t \lambda, \omega=\Delta t \mu, \Phi=\delta u_{n+k}+\delta u_{n+k-1}$

Or

$\alpha_{k} u_{n+k}+\alpha_{k-1} u_{n+k-1}+\frac{1}{(1+\delta)} \sum_{j=0}^{k-2} \alpha_{j} u_{n+j}=\frac{z}{(1+\delta)} \sum_{j=0}^{k} \beta_{j} u_{n+j}+\frac{\omega}{(1+\delta)} \sum_{j=0}^{k-1} \beta_{j}^{*} u_{n+j}$

And

$\Phi+\sum_{j=0}^{k} \alpha_{j} u_{n+j}=z \sum_{j=0}^{k} \beta_{j} u_{n+j}+\omega \sum_{j=0}^{k-1} \beta_{j}^{*} u_{n+j}$,

$z=\Delta t \lambda, \omega=\Delta t \mu \quad, \Phi=\delta u_{n+k}+\delta u_{n+k-1}+\ldots+\delta u_{n}$

Or

$\sum_{j=0}^{k} \alpha_{j} u_{n+j}=\frac{z}{(1+\delta)} \sum_{j=0}^{k} \beta_{j} u_{n+j}+\frac{\omega}{(1+\delta)} \sum_{j=0}^{k-1} \beta_{j}^{*} u_{n+j}$

Introducing polynomials (for more related see [3,4,9]) (from (2.3))

$p(\xi)=\alpha_{k} \xi^{k}+\alpha_{k-1} \xi^{k-1}+\frac{1}{(1+\delta)} \sum_{j=0}^{k-2} \alpha_{j} \xi^{j}$

$\sigma(\xi)=\frac{1}{(1+\delta)} \sum_{j=0}^{k} \beta_{j} \xi^{j}, \sigma^{*}(\xi)=\frac{1}{(1+\delta)} \sum_{j=0}^{k-1} \beta_{j}^{*} \xi^{j}\left(\operatorname{when} \Phi=\delta u_{n+k}+\delta u_{n+k-1}\right)$

Also. we introducing polynomials (from (2.4))

$p(\xi)=\sum_{j=0}^{k} \alpha_{j} \xi^{j}$,

$\sigma(\xi)=\frac{1}{(1+\delta)} \sum_{j=0}^{k} \beta_{j} \xi^{j}, \sigma^{*}(\xi)=\frac{1}{(1+\delta)} \sum_{j=0}^{k-1} \beta_{j}^{*} \xi^{j}\left(\right.$ when $\left.\Phi=\delta u_{n+k}+\delta u_{n+k-1}+\ldots+\delta u_{n}\right)$

And putting $\eta(\xi ; z)=\rho(\xi)-z \sigma(\xi)$

We can write the characteristic equations of the difference equation (2.5) and (2.6) in the form

$$
\eta(\xi ; z)-\omega \sigma^{*}(\xi)=0
$$

And represent the stability region $\boldsymbol{S}$ of method (2.3) and $(2.4)$ as $[5,6]$ 


$$
S=\left\{(z, \omega) \in c^{2}:(2.10) \Rightarrow|\xi|<1\right\}
$$

For example, in the case of the IMEX Euler method (1.2), it follows form $\boldsymbol{u}_{n+1}-\boldsymbol{u}_{n}=\frac{1}{(\mathbf{1}+\delta)} \boldsymbol{u}_{n+1}+\frac{1}{(\mathbf{1}+\delta)} \boldsymbol{u}_{n}$ IMEX Improving Euler( IMEX IEuler) method $\rho(\xi)=\xi-1, \sigma(\xi)=\frac{\xi}{(1+\delta)}, \sigma^{*}(\xi)=\frac{1}{(1+\delta)}$

that the characteristic equation is $\xi-1-\frac{z}{(1+\delta)} \xi-\frac{\omega}{(1+\delta)}=0$

which is rewritten as $\left(1+\frac{\omega}{(1+\delta)}\right) /\left(1-\frac{z}{(1+\delta)}\right)$

hence, the stability region is represented as

$$
S=\left\{(z, \omega) \in C^{2}: 1+\frac{\omega}{(1+\delta)}|<| 1-\frac{z}{(1+\delta)}\right\}
$$

the intersection of the stability region $S$ and the $z$-plane $\{(\boldsymbol{z}, 0): z \in C\}$ is identified with the region $S_{A}=\{z \in C: \eta(\xi ; z)=\mathbf{0} \Rightarrow|\xi|<\mathbf{1}\}$

in the complex, which corresponds to the standard stability region of the implicit formulas. For each $z \in S_{A}$, we denote by $\Gamma_{z}$ the set of all $\omega$ such that (2.7) has a root $|\xi|=\mathbf{1}$. This set is a curve in the complex plane represented in the form

$$
\Gamma_{z}: \frac{\eta(\xi ; z)}{\sigma^{*}(\xi)}, \xi=e^{i \theta}, 0 \leq \theta \leq 2 \pi
$$

Which gives the boundary of the z-section $S \cap\{(\omega, z): \omega \in C\}$

For the IMEX IEuler method, we have

$$
\frac{\eta(\xi ; z)}{\sigma^{*}(\xi)}=\left(-1+\left(1-\frac{z}{(1+\delta)}\right) \xi\right) / \frac{1}{(1+\delta)}
$$

Where $\eta(\xi ; z)=\xi-1-\frac{z}{(1+\delta)} \xi, \sigma^{*}(\xi)=\frac{1}{(1+\xi)}$

Hence, $\Gamma_{z}$ is circle centered at -2 with radius $\left|\mathbf{1}-\frac{\boldsymbol{z}}{(\mathbf{1}+\boldsymbol{\delta})}\right|$ (Fig 1)

Restricting the variable $\mathrm{z}$ on to real line 


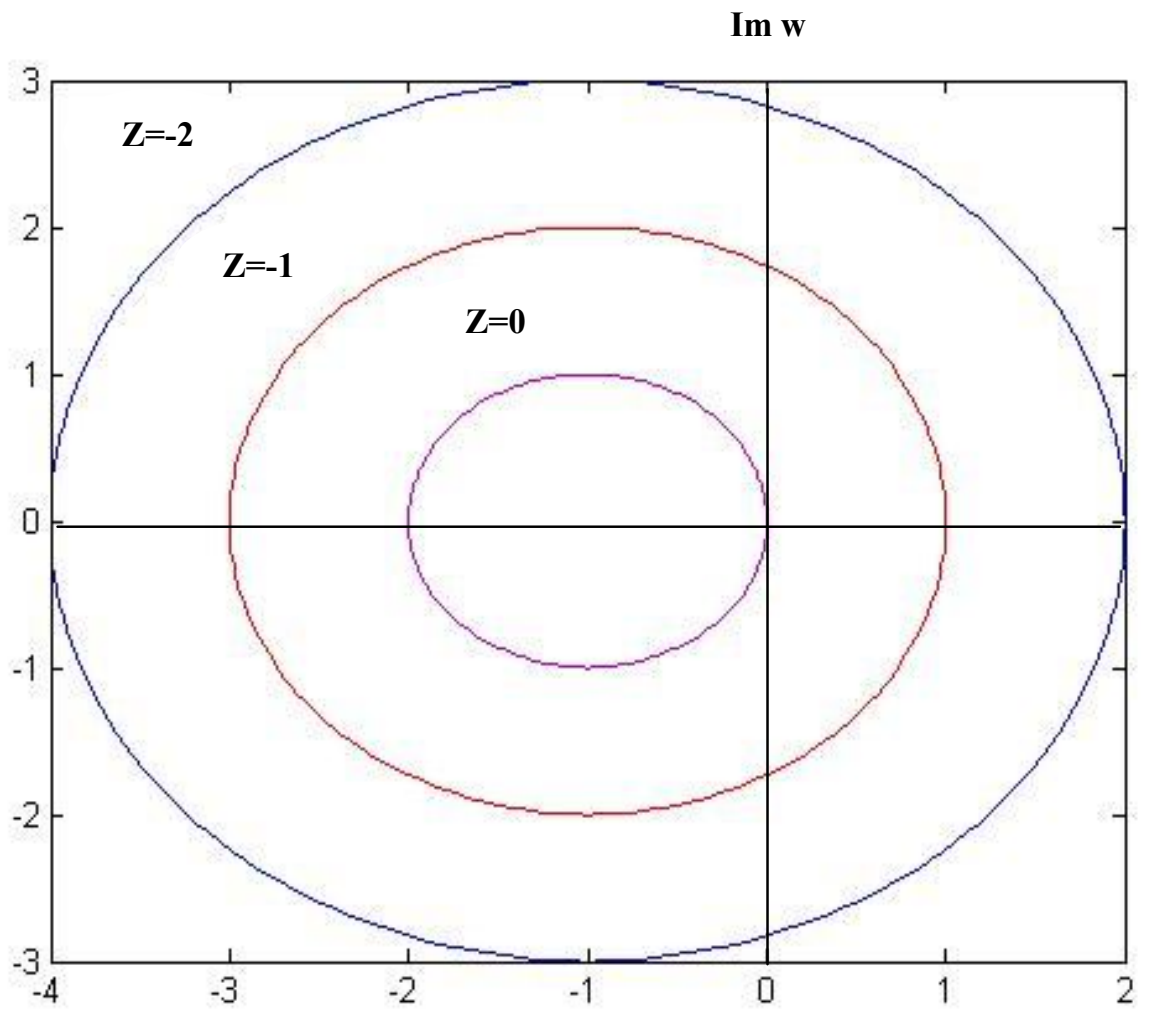

$\operatorname{Re}$

Fig. 1 (a): stability region of the IMEX Euler method

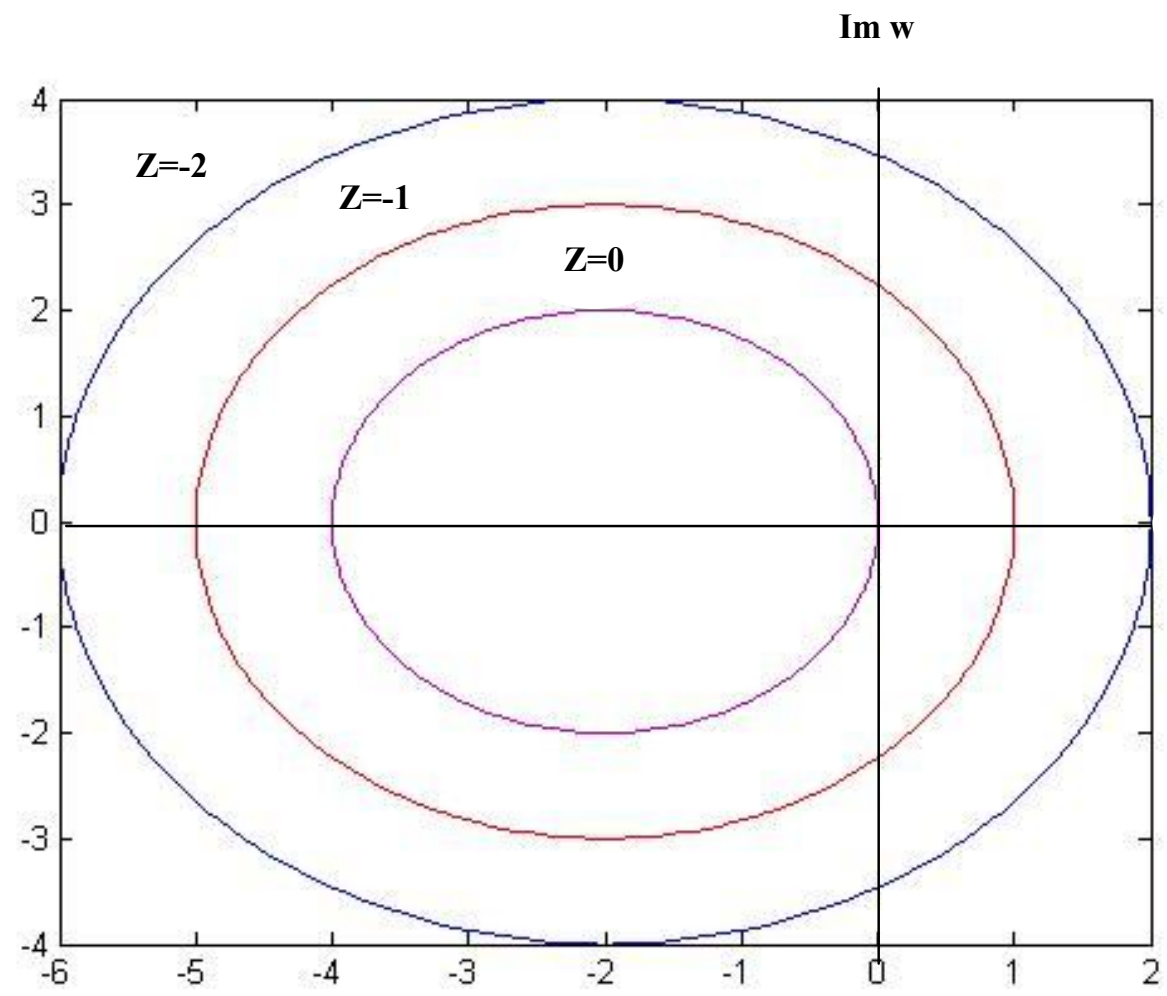

Re w

Fig. 1 (b): stability region of the IMEX IEuler method 
A second-order two-step IMEX method defined with BDF2 (two-step backward differentiation formula)

$$
\begin{aligned}
& \left.\alpha_{2}=3 / 2, \alpha_{1}=-2, \alpha_{0}=1 / 2, \beta_{2}=1, \beta_{1}=\beta_{0}=0\right) \text { and } \\
& \beta_{1}^{*}=2, \beta_{0}^{*}=-1\left(\gamma_{1}=2, \gamma_{0}=-1\right) \\
& \frac{3}{2} u_{n+2}-2 u_{n+1}+\frac{1}{2} u_{n}=\Delta t f\left(t_{n+2}, u_{n+2}\right)+\Delta t\left(2 g\left(t_{n+1}, u_{n+1}\right)-g\left(t_{n}, u_{n}\right)\right)
\end{aligned}
$$

is called (BDF2) method see [5].

This methods have three terms of $\boldsymbol{u}_{n}$, so we discuss the first and second idea.

First: By (2.3) and ( $\boldsymbol{k}=\mathbf{2})$ we have

$$
\begin{aligned}
& (1+\delta)\left(\alpha_{2} u_{n+2}+\alpha_{1} u_{n+1}\right)+\alpha_{0} u_{n}=\Delta t\left(\beta_{2} f\left(t_{n+2}, u_{n+2}\right)+\beta_{1} f\left(t_{n+1}, u_{n+1}\right)+\beta_{0} f\left(t_{n}, u_{n}\right)\right. \\
& \left.+\beta_{1}^{*} g\left(t_{n+1}, u_{n+1}\right)+\beta_{0}^{*} g\left(t_{n}, u_{n}\right)\right)
\end{aligned}
$$

So, from the above condition

$$
(1+\delta)\left(\frac{3}{2} u_{n+2}-2 u_{n+1}\right)+\frac{1}{2} u_{n}=\Delta f f\left(t_{n+2}, u_{n+2}\right)+\Delta t\left(2 g\left(t_{n+1}, u_{n+1}\right)-g\left(t_{n}, u_{n}\right)\right)
$$

we have IMEX Improving BDF2(IBDF2) method

$$
\frac{3}{2} u_{n+2}-2 u_{n+1}+\frac{1}{2(1+\delta)} u_{n}=\frac{\Delta t}{(1+\delta)} f\left(t_{n+2}, u_{n+2}\right)+\frac{2 \Delta t}{(1+\delta)} g\left(t_{n+1}, u_{n+1}\right)-\frac{\Delta t}{(1+\delta)} g\left(t_{n}, u_{n}\right)
$$

From (2.5)

$$
\begin{aligned}
& \frac{3}{2} u_{n+2}-2 u_{n+1}+\frac{1}{2(1+\delta)} u_{n}=\frac{z}{(1+\delta)} u_{n+2}+\frac{2 \omega}{(1+\delta)} u_{n+1}-\frac{\omega}{(1+\delta)} u_{n} \\
& , z=\Delta t \lambda, \omega=\Delta t \mu
\end{aligned}
$$

In the case of this method

$$
\rho(\xi)=\frac{3}{2} \xi^{2}-2 \xi+\frac{1}{2(1+\delta)}, \sigma(\xi)=\frac{1}{(1+\delta)} \xi^{2}, \sigma^{*}(\xi)=\frac{1}{(1+\delta)}(2 \xi-1)
$$

From (2.9) we have $\eta(\xi, z)=\frac{3}{2} \xi^{2}-2 \xi+\frac{1}{2(1+\delta)}-\frac{z}{(1+\delta)} \xi^{2}$ and we denote by $\Gamma_{z}$ the set of all $\omega$ such that (2.10) has a real with $|\xi|=\mathbf{1}$

$$
\Gamma_{z}: \frac{\eta(\xi ; z)}{\sigma^{*}(\xi)}=\frac{\frac{3}{2} \xi^{2}-2 \xi+\frac{1}{2(1+\delta)}-\frac{z}{(1+\delta)} \xi^{2}}{\frac{1}{(1+\delta)}(2 \xi-1)}=\frac{3(1+\delta) \xi^{2}-4(1+\delta) \xi+1-2 z \xi^{2}}{2(2 \xi-1)}
$$

For negative $\mathrm{z}$ is simple closed curve as show in $\operatorname{Fig}(2)$.

In this idea, the parameters $\delta$ appears in left (assume $\delta_{1}=\delta, 0 \geq\left|\delta_{1}\right|<\mathbf{1}$ ) and right hands of IBDF2. 


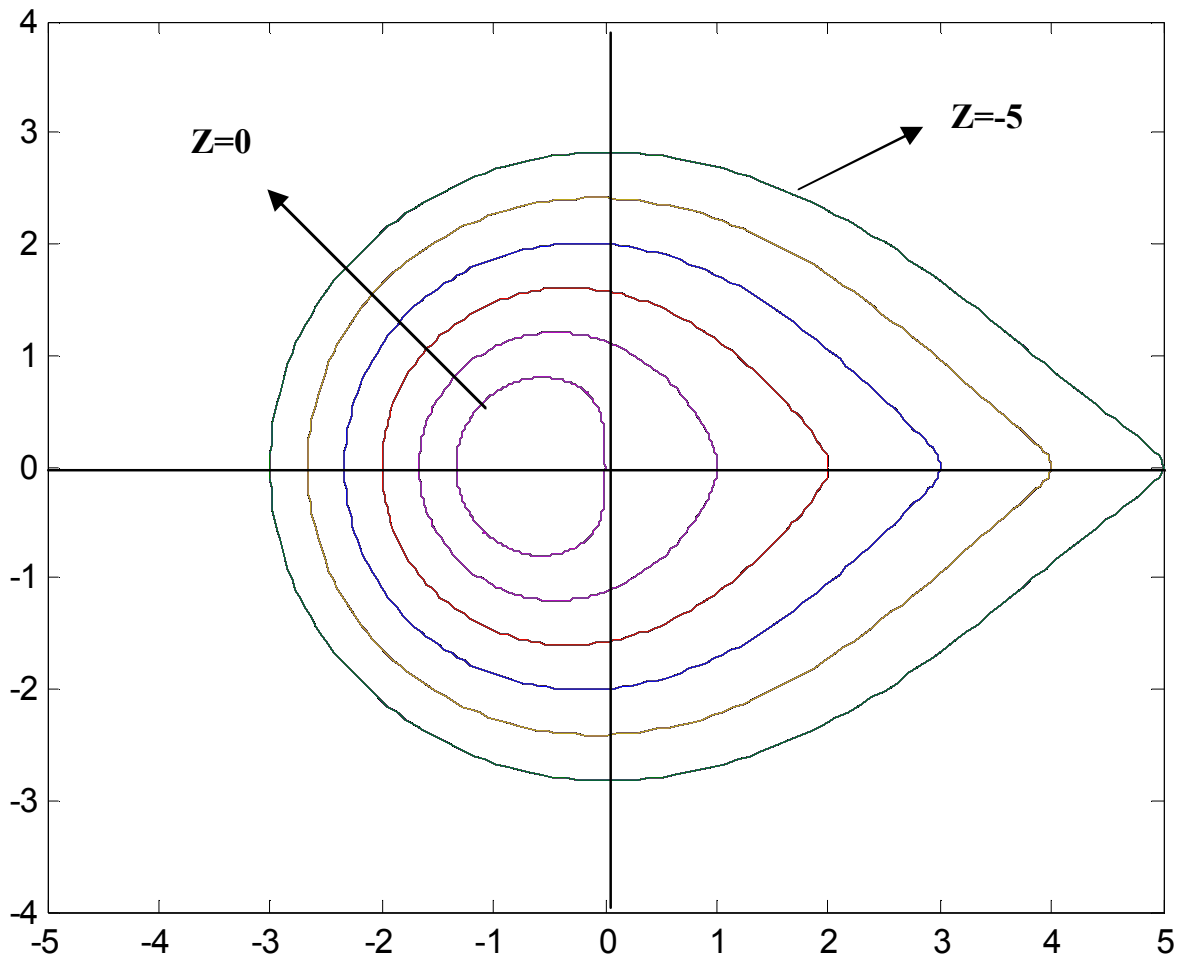

$\operatorname{Re} \mathbf{w}$

Fig. 2 (a): stability region of the IMEX BDF2 method

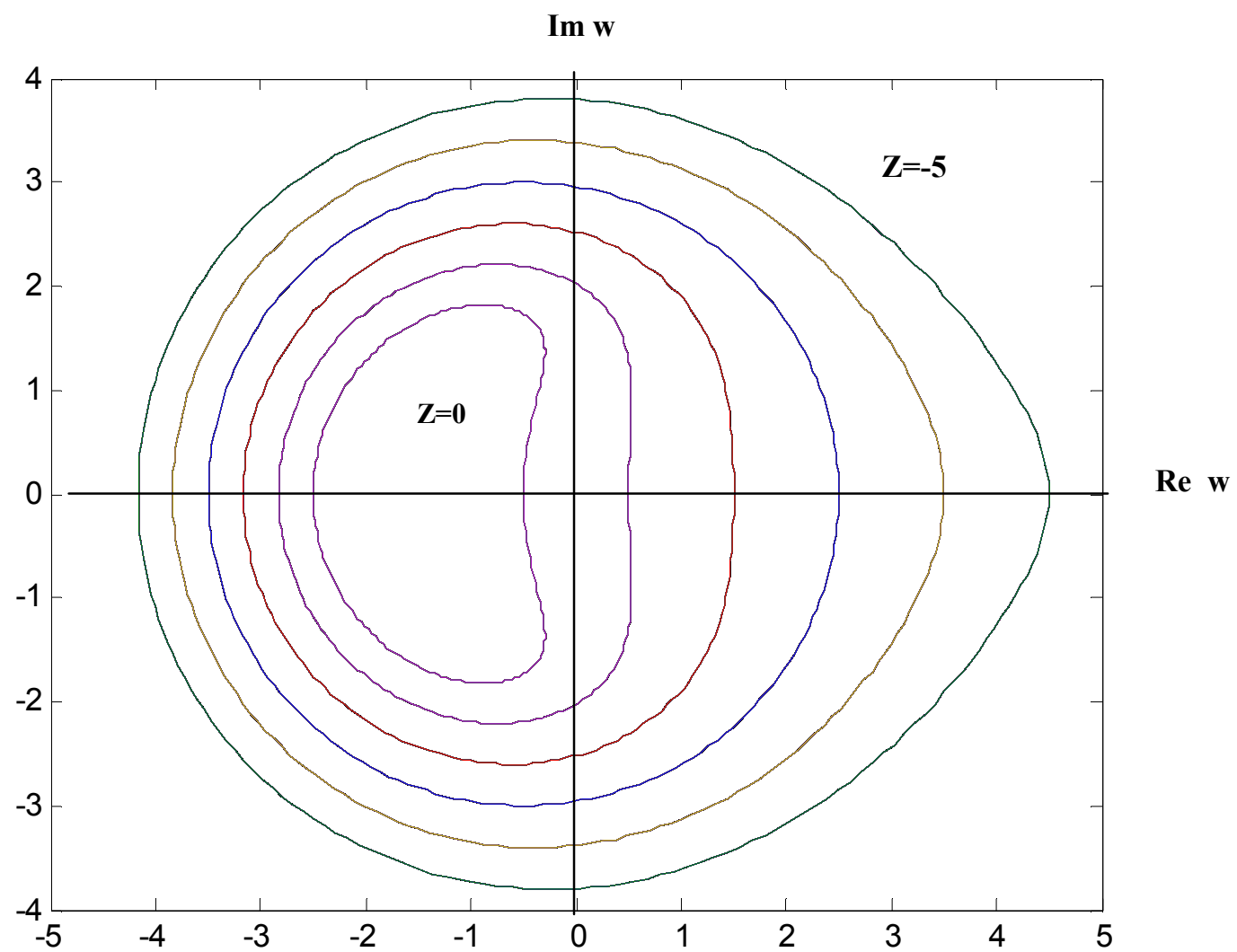

Fig. 2 (b): stability region of the IMEX IBDF2 method 
Second: same as first idea, by (2.4) we have (IMEX IBDF2 method) $\frac{3}{2} u_{n+2}-2 u_{n+1}+\frac{1}{2} u_{n}=\frac{1}{(1+\delta)} f\left(t_{n+2}, u_{n+2}\right)+\frac{2}{(1+\delta)} g\left(t_{n+1}, u_{n+1}\right)-\frac{1}{(1+\delta)} g\left(t_{n}, u_{n}\right)$

From (2.6)

$\frac{3}{2} u_{n+2}-2 u_{n+1}+\frac{1}{2} u_{n}=\frac{z}{(1+\delta)} u_{n+2}+\frac{2 \omega}{(1+\delta)} u_{n+1}-\frac{\omega}{(1+\delta)} u_{n}$

$z=\Delta t \lambda, \omega=\Delta t \mu$

In the case of this method

$\rho(\xi)=\frac{3}{2} \xi^{2}-2 \xi+\frac{1}{2}, \sigma(\xi)=\frac{1}{(1+\delta)} \xi^{2}, \sigma^{*}(\xi)=\frac{1}{(1+\delta)}(2 \xi-1)$

From (2.9) we have $\eta(\xi, z)=\frac{\mathbf{3}}{\mathbf{2}} \xi^{2}-\mathbf{2} \xi+\frac{\mathbf{1}}{\mathbf{2}}-\frac{\boldsymbol{z}}{(\mathbf{1}+\boldsymbol{\delta})} \xi^{2}$ and we denote

by $\Gamma_{z}$ the set of all $\boldsymbol{\omega}$ such that (2.10) has a real with $|\boldsymbol{\xi}|=\mathbf{1}$

$\Gamma_{z}: \frac{\eta(\xi ; z)}{\sigma^{*}(\xi)}=\frac{\frac{3}{2} \xi^{2}-2 \xi+\frac{1}{2}-\frac{z}{(1+\delta)} \xi^{2}}{\frac{1}{(1+\delta)}(2 \xi-1)}=\frac{3(1+\delta) \xi^{2}-4(1+\delta) \xi+(1+\delta)-2 z \xi^{2}}{2(2 \xi-1)}$

For negative $\mathrm{z}$ is simple closed curve as show in Fig 2(c)

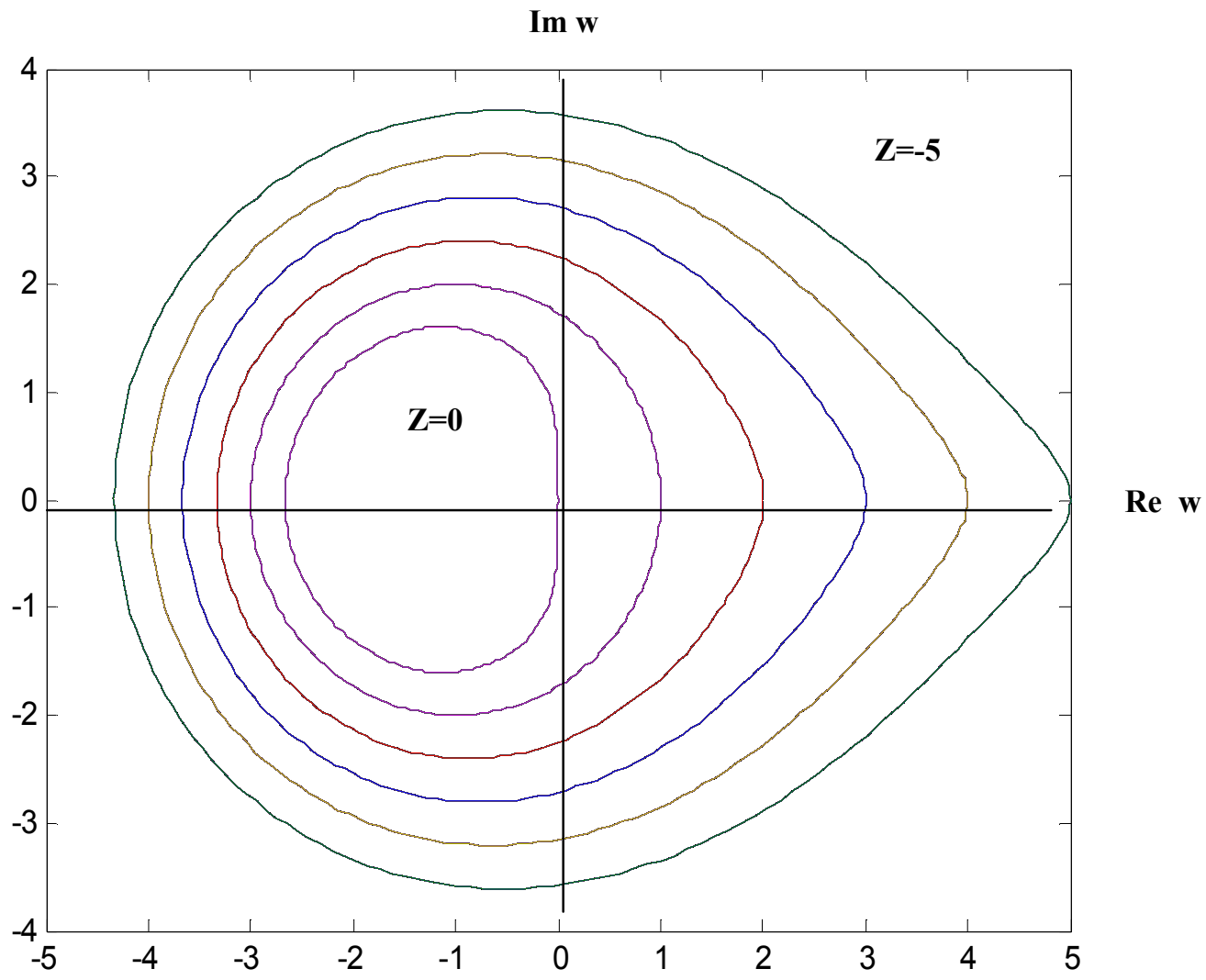

Fig. 2(c) stability region of the IMEX IBDF2 method 
Under condition (1.7) for the step-size, an IMEX linear multi-step method for the DDF (1.8) is defined by

$\Phi+\sum_{j=0}^{k} \alpha_{j} u_{n+j}=\Delta t \sum_{j=0}^{k} \beta_{j} f\left(t_{n+j}, u_{n+j}\right)+\Delta t \sum_{j=0}^{k-1} \beta_{j}^{*} g\left(t_{n+j}, u_{n-m+j}\right)$

Application of the method to the test equation (1.6) yields

$\alpha_{k} u_{n+k}+\alpha_{k-1} u_{n+k-1}+\frac{1}{(1+\delta)} \sum_{j=0}^{k-2} \alpha_{j} u_{n+j}=\frac{z}{(1+\delta)} \sum_{j=0}^{k} \beta_{j} u_{n+j}+\frac{\omega}{(1+\delta)} \sum_{j=0}^{k-1} \beta_{j}^{*} u_{n-m+j}$

$z=\Delta t \lambda, \Delta t \mu, \Phi=\delta u_{n+k}+\delta u_{n+k-1}$

Or

$\sum_{j=0}^{k} \alpha_{j} u_{n+j}=\frac{z}{(1+\delta)} \sum_{j=0}^{k} \beta_{j} u_{n+j}+\frac{\omega}{(1+\delta)} \sum_{j=0}^{k-1} \beta_{j}^{*} u_{n-m+j}$

$z=\Delta t \lambda, \Delta t \mu, \Phi=\delta u_{n+k}+\delta u_{n+k-1}+\ldots+\delta u_{n}$

And the characteristic equation of the difference equation (2.15) and (2.16) is written in the form $\xi^{m} \eta(\xi ; z)-\omega \sigma^{*}(\xi)=0$

Using this equation, we define the $\boldsymbol{p}$-stability region $\boldsymbol{S}_{\boldsymbol{P}}$ of the IMEX

method as [10] $S_{P}=\underset{m \geq 0}{\cap} S_{P}^{(m)}, S_{P}^{(m)}=\left\{(z, \omega) \in C^{2}:(2.13) \Rightarrow|\xi|<1\right\}$

\section{3- Analysis of $\boldsymbol{p}$-stability regions}

Toshiyuki [10] try to find a second-order IMEX method with larger $\boldsymbol{S}$ by the construction of a scheme with larger $S_{P}$ than that of the IMEX BDF2 method.

Furthermore, define the curve $\Gamma^{*}$ in the complex plane by

$\Gamma^{*}: \frac{\sigma^{*}(\xi)}{\sigma(\xi)}, \xi=e^{i \theta}, 0 \leq \theta \leq 2 \pi$

and put $r=\sup \left\{|\omega|: \omega \in \Gamma^{*}\right\}$

Then, have $\boldsymbol{r} \geq \mathbf{1}$.

Using the form of $\Phi$ will not effect for the results of equations (2.19) and (2.20), but reusing $\Gamma_{z}$ will extend the stability region.

For any second order two-step linear multi-step method is represented in

$$
\alpha_{2}=a, \alpha_{1}=1-2 a, \alpha_{0}=a-1
$$

the form [10]

$$
\beta_{2}=b, \beta_{1}=\frac{1}{2}=a-2 b, \beta_{0} \frac{1}{2}-a+b
$$

With the real parameters $\boldsymbol{a}, \boldsymbol{b}$ and conditions $\boldsymbol{a}>\frac{\mathbf{1}}{\mathbf{2}}, \boldsymbol{b}>\frac{\boldsymbol{a}}{\mathbf{2}}$, since $\rho(\xi)$ is written as $\rho(\xi)=(a \xi+1-a)(\xi-1)$

Moreover, it follows form $\gamma_{1}=2, \gamma_{0}=-1$ that $\beta_{1}^{*}=\frac{1}{2}+a, \beta_{0}^{*}=\frac{1}{2}-a$ 
And [10] shows that the supremum $\boldsymbol{r}$ (when $\boldsymbol{a}=\boldsymbol{b}$ ) is written as $\boldsymbol{r}=\frac{\mathbf{2 a}+\mathbf{1}}{\mathbf{2 a - 1}}$, moreover, when taking $\boldsymbol{a}=\boldsymbol{b}=\mathbf{2 0}$ [10] obtain $\boldsymbol{r}=\mathbf{1 . 0 5 1}$ and $\Gamma_{z}$ for much larger stability region (stabilized second-order method) than of the IMEX BDF2 method.

Her, we show that under (2.3) and (2.4) method we obtain the method more larger than that (stabilized second-order method).

By (2.3) we have

$$
\begin{aligned}
& a u_{n+2}+(1-2 a) u_{n+1}+\frac{(a-1)}{(1+\delta)} u_{n}=\frac{b}{(1+\delta)} f\left(t_{n+2}, u_{n+2}\right)+\frac{\left(\frac{1}{2}+a-2 b\right)}{(1+\delta)} f\left(t_{n+1}, u_{n+1}\right)+ \\
& +\frac{\left(\frac{1}{2}-a+b\right)}{(1+\delta)} f\left(t_{n}, u_{n}\right)+\frac{\left(\frac{1}{2}+a\right)}{(1+\delta)} g\left(t_{n+1}, u_{n+1}\right)+\frac{\left(\frac{1}{2}-a\right)}{(1+\delta)} g\left(t_{n}, u_{n}\right)
\end{aligned}
$$

From (2.5)

$$
\begin{aligned}
& a u_{n+2}+(1-2 a) u_{n+1}+\frac{(a-1)}{(1+\delta)} u_{n}=\frac{z b}{(1+\delta)} u_{n+2}+\frac{z\left(\frac{1}{2}+a-2 b\right)}{(1+\delta)} u_{n+1}+\frac{z\left(\frac{1}{2}-a+b\right)}{(1+\delta)} u_{n} \\
& +\frac{\omega\left(\frac{1}{2}+a\right)}{(1+\delta)} u_{n+1}+\frac{\omega\left(\frac{1}{2}-a\right)}{(1+\delta)} u_{n}, z=\Delta t \lambda, \omega=\Delta t \mu
\end{aligned}
$$

In the case of this method

$$
\begin{aligned}
\rho(\xi) & =a \xi^{2}+(1-2 a) \xi+\frac{(a-1)}{(1+\delta)} \\
\sigma(\xi) & =\frac{b}{(1+\delta)} \xi^{2}+\frac{(0.5+a-2 b)}{(1+\delta)} \xi+\frac{(0.5-a+b)}{(1+\delta)} \\
\sigma^{*}(\xi) & =\frac{(0.5+a)}{(1+\delta)} \xi+\frac{(0.5-a)}{(1+\delta)}
\end{aligned}
$$

So,

$$
\begin{aligned}
& \Gamma_{z}: \frac{\eta(\xi ; z)}{\sigma^{*}(\xi)}=\frac{a \xi^{2}+(1-2 a) \xi+\frac{(a-1)}{(1+\delta)}-\frac{z}{(1+\delta)}\left(b \xi^{2}+(0.5+a-2 b) \xi+(0.5-a+b)\right)}{\frac{1}{(1+\delta)}((0.5+a) \xi+(0.5-a))} \\
& =\frac{a(1+\delta) \xi^{2}+(1-2 a)(1+\delta) \xi+(a-1)-z\left(b \xi^{2}+(0.5+a-2 b) \xi+(0.5-a+b)\right)}{(0.5+a) \xi+(0.5-a)}
\end{aligned}
$$

Now, by (2.4) we have 


$$
\begin{aligned}
& a u_{n+2}+(1-2 a) u_{n+1}+(a-1) u_{n}=\frac{b}{(1+\delta)} f\left(t_{n+2}, u_{n+2}\right)+\frac{\left(\frac{1}{2}+a-2 b\right)}{(1+\delta)} f\left(t_{n+1}, u_{n+1}\right)+ \\
& +\frac{\left(\frac{1}{2}-a+b\right)}{(1+\delta)} f\left(t_{n}, u_{n}\right)+\frac{\left(\frac{1}{2}+a\right)}{(1+\delta)} g\left(t_{n+1}, u_{n+1}\right)+\frac{\left(\frac{1}{2}-a\right)}{(1+\delta)} g\left(t_{n}, u_{n}\right)
\end{aligned}
$$

From (2.6)

$$
\begin{aligned}
& a u_{n+2}+(1-2 a) u_{n+1}+(a-1) u_{n}=\frac{z b}{(1+\delta)} u_{n+2}+\frac{z\left(\frac{1}{2}+a-2 b\right)}{(1+\delta)} u_{n+1}+\frac{z\left(\frac{1}{2}-a+b\right)}{(1+\delta)} u_{n} \\
& +\frac{\omega\left(\frac{1}{2}+a\right)}{(1+\delta)} u_{n+1}+\frac{\omega\left(\frac{1}{2}-a\right)}{(1+\delta)} u_{n}, z=\Delta t \lambda, \omega=\Delta t \mu
\end{aligned}
$$

In the case of this method

$$
\begin{aligned}
& \rho(\xi)=a \xi^{2}+(1-2 a) \xi+(a-1), \sigma(\xi)=\frac{b}{(1+\delta)} \xi^{2}+\frac{(0.5+a-2 b)}{(1+\delta)} \xi+\frac{(0.5-a+b)}{(1+\delta)} \\
& \sigma^{*}(\xi)=\frac{(0.5+a)}{(1+\delta)} \xi+\frac{(0.5-a)}{(1+\delta)} \\
& \Gamma_{z}: \frac{\eta(\xi ; z)}{\sigma^{*}(\xi)}=\frac{a \xi^{2}+(1-2 a) \xi+(a-1)-\frac{z}{(1+\delta)}\left(b \xi^{2}+(0.5+a-2 b) \xi+(0.5-a+b)\right)}{\frac{1}{(1+\delta)}((0.5+a) \xi+(0.5-a))} \\
& =\frac{a(1+\delta) \xi^{2}+(1-2 a)(1+\delta) \xi+(a-1)(1+\delta)-z\left(b \xi^{2}+(0.5+a-2 b) \xi+(0.5-a+b)\right)}{(0.5+a) \xi+(0.5-a)}
\end{aligned}
$$

\section{3- Numerical results}

Here we show by numerical examples [9] the performance of the improved methods. From the coming tables we show the stability behavior of the normal methods and improved methods by using different values for the step size $\boldsymbol{h}$.

These values, of $\boldsymbol{h}$ showed the stability behavior of the newly developed methods.

We include test results of the following problems for the IMEX Euler method and IMEX second two-step method also IMEX second two-step method with $\mathrm{a}=\mathrm{b}=20$.

Example-1: $y^{\prime \prime}+1001 y^{\prime}+1000 y, y(0)=1, y^{\prime}(0)=-1$

$$
y(x)=e^{-x}+e^{-1000}
$$

Example-2: $\varepsilon y^{\prime \prime}+y^{\prime}=0, y(0)=1, y(1)=2$

$$
y(x)=\frac{2-\exp (-1 / \varepsilon)-\exp (-x / \varepsilon)}{1-\exp (-1 / \varepsilon}
$$


Example-3: $\left.y^{\prime \prime}+21 y^{\prime}+20 y, y(0)=1, y^{\prime} 0\right)=-1$ $y(x)=e^{-x}+e^{-20}$

Table (1) and (2) displays the results with the IMEX Euler and IMEX IEuler methods. We can see that the method (IMEX IEuler) produce stable solutions with much larger $\boldsymbol{h}$ than that IMEX Euler method.

Also, with the IMEX IBDF2 and IMEX BDF2 (see tables 3-10) indicate the effect of step size $\boldsymbol{h}$ which lies outside of the stability region of IMEX BDF2 method and inside for the IMEX IBDF2 method.

On the other hand, the IMEX IBDF2 method with second case is more stable solution than the IMEX IBDF2 method with first case. But, when taken $\delta_{1}$ differences from $\delta$ we have stability results.

\section{4- conclusions}

In this paper we discuss two idea and we see that these methods( IMEX Improving Euler method and IMEX Improving BDF2method) its more stability than the methods developed by Toshiyuki KOTO( IMEX Euler method and IMEX BDF2method).

\section{References}

1) Ascher UM, Ruuth SJ Wetton BTR. (1995) Implict-explicit methods for time-dependent PDF $_{\mathrm{s}}$. SIAM J Numer Anal, 32:797823

2) Bakke VL, Jackiewicz Z. (1986) Stability analysis of linear muttistep methods for delay differential equations. Internal. J. Math. Sci., Vol.9, No.3:447-458.

3) Ernst Hairer. (2008) Conjugate-simplicity of linear multistep methods. Journal of Computational Mathematics, Vol.26, No.5, 657-659.

4) Ferreira JC, Meira M, Meneguette M, Nogueira JR. (2008) Linear multistep methods and order stars: some properties. TEMA Tend. Mat Apl. Comput., 9 No.2, 233-242.

5) Frank J, Hundsdorfer W, Verwer JG. On the stability of implicitexplicit linear multistep methods. Appl Numer Math,1997, 25:193205.

6) Hundsdorfer HW. (1996) Numerical solution of advection-diffusion equations. CWI Report NM-N9603.

7) Hundsdorfer W. (1996) Stability results for implicit-explicit linear 2-step methods. CWI, P. O. Box 94079, 1090 GB Amsterdam.

8) Jain MK, Iyengar SRK, Jain RK.(1989) Numerical methods for scientific and engineering computation.

9) Lambert J.D. (1974) computational methods in ordinary differential equations. John Wiley and Sons Ltd.

10) Toshiyuki KOTO. (2009) Stability of implicit-explicit linear multistep methods for ordinary and delay differential equations. Front. Math. China, 4(1):113-129. 
Step size $=\mathbf{0 . 0 5}$

$$
y^{\prime \prime}+21 y^{\prime}+20 y=0
$$

\begin{tabular}{|l|l|l|l|l|l|}
\hline$X$ & Theoretical solution & $\begin{array}{l}\text { Numerical solution of } \\
\text { IEU method }\end{array}$ & Error of IEU method & $\begin{array}{l}\text { Numerical solution of } \\
\text { EU method }\end{array}$ & Error of EU method \\
\hline 0.05 & 1.319108865672156 & 1.812222222222222 & 0.493113356550066 & 2.052500000000000 & 0.733391134327844 \\
\hline 1 & 0.367879443232596 & 0.585178006474125 & 0.217298563241529 & 0.100556402773683 & 0.468435846006279 \\
\hline 2 & 0.135335283236613 & 0.154523051570657 & 0.019187768334045 & 2.901709408161799 & 3.037044691398412 \\
\hline 3 & 0.049787068367864 & 0.040803607301091 & 0.008983461066773 & 21.663314171561830 & 21.713101239929692 \\
\hline 4 & 0.018315638888734 & 0.010774666639432 & 0.007540972249302 & $1.595809859695625 \mathrm{e}+02$ & $1.595993016084512 \mathrm{e}+02$ \\
\hline 5 & 0.006737946999085 & 0.002845175926095 & 0.003892771072991 & $1.175251826606274 \mathrm{e}+03$ & $1.175258564553273 \mathrm{e}+03$ \\
\hline 6 & 0.002478752176666 & $7.513017637876931 \mathrm{e}-04$ & 0.001727450412879 & $8.655232990428390 \mathrm{e}+03$ & $8.655235469180567 \mathrm{e}+03$ \\
\hline 7 & $9.118819655545162 \mathrm{e}-04$ & $1.983899607379422 \mathrm{e}-04$ & $7.134920048165740 \mathrm{e}-04$ & $6.374212758587310 \mathrm{e}+04$ & $6.374212849775506 \mathrm{e}+04$ \\
\hline 8 & $3.354626279025119 \mathrm{e}-04$ & $5.238717439338315 \mathrm{e}-05$ & $2.830754535091287 \mathrm{e}-04$ & $4.694337896421047 \mathrm{e}+05$ & $4.694337899775673 \mathrm{e}+05$ \\
\hline 9 & $1.234098040866796 \mathrm{e}-04$ & $1.383344212940241 \mathrm{e}-05$ & $1.095763619572772 \mathrm{e}-04$ & $3.457181164130893 \mathrm{e}+06$ & $3.457181164254303 \mathrm{e}+06$ \\
\hline 10 & $4.539992976248485 \mathrm{e}-05$ & $3.652881136717617 \mathrm{e}-06$ & $4.174704862576723 \mathrm{e}-05$ & $2.546067595757721 \mathrm{e}+07$ & $2.546067595762261 \mathrm{e}+07$ \\
\hline
\end{tabular}

Table (1) : Numerical results of IEU ( $\delta=0.5)$ method and EU method . 


\section{Step size $=\mathbf{0 . 0 1 5}$}

$$
\varepsilon y^{\prime \prime}+y^{\prime}=0, \varepsilon=0.01
$$

\begin{tabular}{|c|c|c|c|c|c|}
\hline $\mathrm{X}$ & Theoretical solution & $\begin{array}{l}\text { Numerical solution } \\
\text { of IEU method }\end{array}$ & Error of IEU method & $\begin{array}{l}\text { Numerical solution of EU } \\
\text { method }\end{array}$ & Error of EU method \\
\hline 0.015 & 1.776869839851570 & 1.996093750000000 & 0.223130160148430 & 1.750000000000000 & 0.026869839851570 \\
\hline 0.15 & 1.999999694097679 & 2.001174444536446 & 0.001174750438766 & 130 & $1.280000003059023 \mathrm{e}+02$ \\
\hline 0.30 & 1.999999999999907 & 2.000308967673098 & $3.089676731915514 \mathrm{e}-04$ & 131074 & 131072 \\
\hline 0.45 & 2 & 2.000081281848617 & $8.128184861666554 \mathrm{e}-05$ & 134217730 & 134217728 \\
\hline 0.60 & 2 & 2.000021383269157 & $2.138326915712696 \mathrm{e}-05$ & $1.374389534740000 \mathrm{e}+11$ & $1.374389534720000 \mathrm{e}+11$ \\
\hline 0.75 & 2 & 2.000005625415855 & $5.625415854471072 \mathrm{e}-06$ & $1.407374883553300 \mathrm{e}+14$ & $1.407374883553280 \mathrm{e}+14$ \\
\hline 0.90 & 2 & 2.000001479909518 & $1.479909518042888 \mathrm{e}-06$ & $1.441151880758559 \mathrm{e}+17$ & $1.441151880758559 \mathrm{e}+17$ \\
\hline 1.05 & 2 & 2.000000389328050 & $3.893280497990759 \mathrm{e}-07$ & $1.475739525896764 \mathrm{e}+20$ & $1.475739525896764 \mathrm{e}+20$ \\
\hline 1.20 & 2 & 2.000000102422702 & $1.024227018753265 \mathrm{e}-07$ & $1.511157274518287 \mathrm{e}+23$ & $1.511157274518287 \mathrm{e}+23$ \\
\hline 1.35 & 2 & 2.000000026944912 & $2.694491163524049 \mathrm{e}-08$ & $1.547425049106725 \mathrm{e}+26$ & $1.547425049106725 \mathrm{e}+26$ \\
\hline 1.50 & 2 & 1.999999991898803 & $8.101197268572946 \mathrm{e}-09$ & $1.584563250285287 \mathrm{e}+29$ & $1.584563250285287 \mathrm{e}+29$ \\
\hline 1.65 & 2 & 2.000000001864825 & $1.864824739072901 \mathrm{e}-09$ & $1.622592768292134 \mathrm{e}+32$ & $1.622592768292134 \mathrm{e}+32$ \\
\hline 1.80 & 2 & 2.000000000490590 & $4.905902351026725 \mathrm{e}-10$ & $1.661534994731145 \mathrm{e}+35$ & $1.661534994731145 \mathrm{e}+35$ \\
\hline 1.95 & 2 & 2.000000000129063 & $1.290625384342548 \mathrm{e}-10$ & $1.701411834604692 \mathrm{e}+38$ & $1.701411834604692 \mathrm{e}+38$ \\
\hline
\end{tabular}

Table (2) : Numerical results of IEU ( $\delta=0.5)$ method and EU method . 


\section{Step size $=0.1$ (First case)}

$$
y^{\prime \prime}+21 y^{\prime}+20 y=0
$$

\begin{tabular}{|l|l|l|l|l|l|}
\hline$X$ & Theoretical solution & $\begin{array}{l}\text { Numerical solution of } \\
\text { IBDF2 method }\end{array}$ & Error of IBDF2 method & $\begin{array}{l}\text { Numerical solution of } \\
\text { BDF2 method }\end{array}$ & Error of BDF2 method \\
\hline 0.1 & 1.040172701272572 & 0.864864864864865 & 0.175307836407707 & 0.100000000000000 & 1.140172701272572 \\
\hline 4 & 0.018315638888734 & 0.014309507522831 & 0.004006131365903 & $3.400074047538720 \mathrm{e}+05$ & $3.400073864382331 \mathrm{e}+05$ \\
\hline 8 & $3.354626279025119 \mathrm{e}-04$ & $2.081560443943902 \mathrm{e}-05$ & $3.562782323419509 \mathrm{e}-04$ & $1.359807673864999 \mathrm{e}+14$ & $1.359807673864999 \mathrm{e}+14$ \\
\hline 12 & $6.144212353328210 \mathrm{e}-06$ & $1.409269523871745 \mathrm{e}-05$ & $7.948482885389243 \mathrm{e}-06$ & $5.438359010233444 \mathrm{e}+22$ & $5.438359010233444 \mathrm{e}+22$ \\
\hline 16 & $1.125351747192591 \mathrm{e}-07$ & $3.979789108786082 \mathrm{e}-07$ & $5.105140855978673 \mathrm{e}-07$ & $2.174994985880914 \mathrm{e}+31$ & $2.174994985880914 \mathrm{e}+31$ \\
\hline 20 & $2.061153622438558 \mathrm{e}-09$ & $1.332680337402335 \mathrm{e}-08$ & $1.126564975158479 \mathrm{e}-08$ & $8.698585694150505 \mathrm{e}+39$ & $8.698585694150505 \mathrm{e}+39$ \\
\hline 24 & $3.775134544279098 \mathrm{e}-11$ & $2.875186713547607 \mathrm{e}-10$ & $3.252700167975517 \mathrm{e}-10$ & $3.478876667287276 \mathrm{e}+48$ & $3.478876667287276 \mathrm{e}+48$ \\
\hline 28 & $6.914400106940203 \mathrm{e}-13$ & $3.956601835756914 \mathrm{e}-12$ & $3.265161825062894 \mathrm{e}-12$ & $1.391327658510566 \mathrm{e}+57$ & $1.391327658510566 \mathrm{e}+57$ \\
\hline 32 & $1.266416554909418 \mathrm{e}-14$ & $7.035453149140475 \mathrm{e}-14$ & $5.769036594231058 \mathrm{e}-14$ & $5.564418743381209 \mathrm{e}+65$ & $5.564418743381209 \mathrm{e}+65$ \\
\hline 36 & $2.319522830243570 \mathrm{e}-16$ & $7.542522073258293 \mathrm{e}-15$ & $7.774474356282650 \mathrm{e}-15$ & $2.225410798261433 \mathrm{e}+74$ & $2.225410798261433 \mathrm{e}+74$ \\
\hline 40 & $4.248354255291589 \mathrm{e}-18$ & $3.517011080835734 \mathrm{e}-16$ & $3.474527538282818 \mathrm{e}-16$ & $8.900216625338383 \mathrm{e}+82$ & $8.900216625338383 \mathrm{e}+82$ \\
\hline
\end{tabular}

Table (3) : Numerical results of IBDF2 $\left(\delta=0.4, \delta_{1}=-0.45\right)$ method and BDF2 method . 


\section{Step size $=0.1($ Second case $)$ \\ $y^{\prime \prime}+21 y^{\prime}+20 y=0$}

\begin{tabular}{|c|c|c|c|c|c|}
\hline $\mathrm{X}$ & Theoretical solution & $\begin{array}{l}\text { Numerical solution of } \\
\text { IBDF2 method }\end{array}$ & Error of IBDF2 method & $\begin{array}{l}\text { Numerical solution of } \\
\text { BDF2 method }\end{array}$ & Error of BDF2 method \\
\hline 0.1 & 1.040172701272572 & 0.950000000000000 & 0.090172701272572 & 0.100000000000000 & 1.140172701272572 \\
\hline 4 & 0.018315638888734 & 0.018194231095980 & $1.214077927545534 \mathrm{e}-04$ & $3.400074047538720 \mathrm{e}+05$ & $3.400073864382331 \mathrm{e}+05$ \\
\hline 8 & $3.354626279025119 \mathrm{e}-04$ & $3.484707698997363 \mathrm{e}-04$ & $1.300814199722448 \mathrm{e}-05$ & $1.359807673864999 \mathrm{e}+14$ & $1.359807673864999 \mathrm{e}+14$ \\
\hline 12 & $6.144212353328210 \mathrm{e}-06$ & $6.700293521008944 \mathrm{e}-06$ & $5.560811676807337 \mathrm{e}-07$ & $5.438359010233444 \mathrm{e}+22$ & $5.438359010233444 \mathrm{e}+22$ \\
\hline 16 & $1.125351747192591 \mathrm{e}-07$ & $1.259208698742138 \mathrm{e}-07$ & $1.338569515495468 \mathrm{e}-08$ & $2.174994985880914 \mathrm{e}+31$ & $2.174994985880914 \mathrm{e}+31$ \\
\hline 20 & $2.061153622438558 \mathrm{e}-09$ & $2.347232716461780 \mathrm{e}-09$ & $2.860790940232218 \mathrm{e}-10$ & $8.698585694150505 e+39$ & $8.698585694150505 e+39$ \\
\hline 24 & $3.775134544279098 \mathrm{e}-11$ & $4.372019349018589 \mathrm{e}-11$ & $5.968848047394910 \mathrm{e}-12$ & $3.478876667287276 \mathrm{e}+48$ & $3.478876667287276 \mathrm{e}+48$ \\
\hline 28 & $6.914400106940203 \mathrm{e}-13$ & $8.147436755353200 \mathrm{e}-13$ & $1.233036648412997 \mathrm{e}-13$ & $1.391327658510566 \mathrm{e}+57$ & $1.391327658510566 \mathrm{e}+57$ \\
\hline 32 & $1.266416554909418 \mathrm{e}-14$ & $1.518675543002552 \mathrm{e}-14$ & $2.522589880931347 \mathrm{e}-15$ & $5.564418743381209 \mathrm{e}+65$ & $5.564418743381209 \mathrm{e}+65$ \\
\hline 36 & $2.319522830243570 \mathrm{e}-16$ & $2.830907683035232 \mathrm{e}-16$ & $5.113848527916624 \mathrm{e}-17$ & $2.225410798261433 \mathrm{e}+74$ & $2.225410798261433 \mathrm{e}+74$ \\
\hline 40 & $4.248354255291589 \mathrm{e}-18$ & $5.276943514714216 \mathrm{e}-18$ & $1.028589259422627 \mathrm{e}-18$ & $8.900216625338383 \mathrm{e}+82$ & $8.900216625338383 \mathrm{e}+82$ \\
\hline
\end{tabular}

Table (4) : Numerical results of IBDF2 $(\delta=1)$ method and BDF2 method . 


\section{Step size $=\mathbf{0 . 0 0 2}$ (First case) \\ $y^{\prime \prime}+1001 y^{\prime}+1000 y=0$}

\begin{tabular}{|l|l|l|l|l|l|}
\hline $\mathrm{X}$ & Theoretical solution & $\begin{array}{l}\text { Numerical solution } \\
\text { of IBDF2 method }\end{array}$ & Error of IBDF2 method & $\begin{array}{l}\text { Numerical solution of } \\
\text { BDF2 method }\end{array}$ & Error of BDF2 method \\
\hline 0.004 & 0.996007989343992 & 0.997334332658333 & 0.001326343314342 & 0.994677328000000 & 0.001330661343991 \\
\hline 0.080 & 0.923116346386636 & 0.924411804988129 & 0.001295458601493 & 3.967458686904738 & 3.044342340518103 \\
\hline 0.160 & 0.852143788966211 & 0.853254089901039 & 0.001110300934828 & $1.192809433611261 \mathrm{e}+07$ & $1.192809348396882 \mathrm{e}+07$ \\
\hline 0.240 & 0.786627861066553 & 0.787573825508518 & $9.459644419644864 \mathrm{e}-04$ & $4.570847161995962 \mathrm{e}+13$ & $4.570847161995883 \mathrm{e}+13$ \\
\hline 0.320 & 0.726149037073691 & 0.726949378816755 & $8.003417430635373 \mathrm{e}-04$ & $1.751549280657117 \mathrm{e}+20$ & $1.751549280657117 \mathrm{e}+20$ \\
\hline 0.400 & 0.670320046035639 & 0.670991572149895 & $6.715261142551388 \mathrm{e}-04$ & $6.711939327305943 \mathrm{e}+26$ & $6.711939327305943 \mathrm{e}+26$ \\
\hline 0.480 & 0.618783391806141 & 0.619341185254219 & $5.577934480777147 \mathrm{e}-04$ & $2.572016102026799 \mathrm{e}+33$ & $2.572016102026799 \mathrm{e}+33$ \\
\hline 0.560 & 0.571209063848815 & 0.571666649289019 & $4.575854402040225 \mathrm{e}-04$ & $9.855969350277760 \mathrm{e}+39$ & $9.855969350277760 \mathrm{e}+39$ \\
\hline 0.640 & 0.527292424043049 & 0.527661918325668 & $3.694942826194536 \mathrm{e}-04$ & $3.776808852676557 \mathrm{e}+46$ & $3.776808852676557 \mathrm{e}+46$ \\
\hline 0.720 & 0.486752255959972 & 0.487044504690633 & $2.922487306610266 \mathrm{e}-04$ & $1.447273687925382 \mathrm{e}+53$ & $1.447273687925382 \mathrm{e}+53$ \\
\hline 0.800 & 0.449328964117222 & 0.449553665540325 & $2.247014231034350 \mathrm{e}-04$ & $5.545954824472313 \mathrm{e}+59$ & $5.545954824472313 \mathrm{e}+59$ \\
\hline 0.880 & 0.414782911681581 & 0.414948729026548 & $1.658173449664013 \mathrm{e}-04$ & $2.125210675195637 \mathrm{e}+66$ & $2.125210675195637 \mathrm{e}+66$ \\
\hline 0.960 & 0.382892885975112 & 0.383007549307375 & $1.146633322627877 \mathrm{e}-04$ & $8.143810321057266 \mathrm{e}+72$ & $8.143810321057266 \mathrm{e}+72$ \\
\hline 1.040 & 0.353454681958780 & 0.353525080485440 & $7.039852666018698 \mathrm{e}-05$ & $3.120709269882304 \mathrm{e}+79$ & $3.120709269882304 \mathrm{e}+79$ \\
\hline
\end{tabular}

Table (5) : Numerical results of IBDF2 ( $\left.\delta=1, \delta_{1}=-\mathbf{0 . 0 0 0 0 5}\right)$ method and BDF2 method . 


\section{Step size $=\mathbf{0 . 0 0 2}$ (Second case) \\ $y^{\prime \prime}+1001 y^{\prime}+1000 y=0$}

\begin{tabular}{|c|c|c|c|c|c|}
\hline $\mathrm{X}$ & Theoretical solution & $\begin{array}{l}\text { Numerical solution } \\
\text { of IBDF } 2 \text { method }\end{array}$ & Error of IBDF2 method & $\begin{array}{l}\text { Numerical solution of } \\
\text { BDF2 method }\end{array}$ & Error of BDF2 method \\
\hline 0.004 & 0.996007989343992 & 0.997335999333333 & 0.001328009989342 & 0.994677328000000 & 0.001330661343991 \\
\hline 0.080 & 0.923116346386636 & 0.924501136035672 & 0.001384789649037 & 3.967458686904738 & 3.044342340518103 \\
\hline 0.160 & 0.852143788966211 & 0.853422225242536 & 0.001278436276324 & $1.192809433611261 \mathrm{e}+07$ & $1.192809348396882 \mathrm{e}+07$ \\
\hline 0.240 & 0.786627861066553 & 0.787808111657584 & 0.001180250591031 & $4.570847161995962 \mathrm{e}+13$ & $4.570847161995883 \mathrm{e}+13$ \\
\hline 0.320 & 0.726149037073691 & 0.727238642768418 & 0.001089605694727 & $1.751549280657117 \mathrm{e}+20$ & $1.751549280657117 \mathrm{e}+20$ \\
\hline 0.400 & 0.670320046035639 & 0.671325968480920 & 0.001005922445280 & $6.711939327305943 \mathrm{e}+26$ & $6.711939327305943 \mathrm{e}+26$ \\
\hline 0.480 & 0.618783391806141 & 0.619712057985838 & $9.286661796972240 \mathrm{e}-04$ & $2.572016102026799 \mathrm{e}+33$ & $2.572016102026799 \mathrm{e}+33$ \\
\hline 0.560 & 0.571209063848815 & 0.572066407146528 & $8.573432977131423 \mathrm{e}-04$ & $9.855969350277760 \mathrm{e}+39$ & $9.855969350277760 \mathrm{e}+39$ \\
\hline 0.640 & 0.527292424043049 & 0.528083922151174 & $7.914981081257322 \mathrm{e}-04$ & $3.776808852676557 \mathrm{e}+46$ & $3.776808852676557 \mathrm{e}+46$ \\
\hline 0.720 & 0.486752255959972 & 0.487482965877312 & $7.307099173398668 \mathrm{e}-04$ & $1.447273687925382 \mathrm{e}+53$ & $1.447273687925382 \mathrm{e}+53$ \\
\hline 0.800 & 0.449328964117222 & 0.450003554458738 & $6.745903415167342 \mathrm{e}-04$ & $5.545954824472313 e+59$ & $5.545954824472313 \mathrm{e}+59$ \\
\hline 0.880 & 0.414782911681581 & 0.415405692506728 & $6.227808251464406 \mathrm{e}-04$ & $2.125210675195637 \mathrm{e}+66$ & $2.125210675195637 \mathrm{e}+66$ \\
\hline 0.960 & 0.382892885975112 & 0.383467836325317 & $5.749503502051057 \mathrm{e}-04$ & $8.143810321057266 \mathrm{e}+72$ & $8.143810321057266 \mathrm{e}+72$ \\
\hline 1.040 & 0.353454681958780 & 0.353985475280021 & $5.307933212408966 \mathrm{e}-04$ & $3.120709269882304 \mathrm{e}+79$ & $3.120709269882304 \mathrm{e}+79$ \\
\hline
\end{tabular}

Table (6) : Numerical results of IBDF2 $(\delta=1)$ method and BDF2 method . 


\section{Step size $=0.015$ (First case) \\ $\varepsilon y^{\prime \prime}+y^{\prime}=0, \varepsilon=0.01$}

\begin{tabular}{|c|c|c|c|c|c|}
\hline $\mathrm{X}$ & Theoretical solution & $\begin{array}{l}\text { Numerical solution of } \\
\text { IBDF } 2 \text { method }\end{array}$ & Error of IBDF2 method & $\begin{array}{l}\text { Numerical solution of } \\
\text { BDF2 method }\end{array}$ & $\begin{array}{l}\text { Error of BDF2 } \\
\text { method }\end{array}$ \\
\hline 0.015 & 1.776869839851570 & 1.750000000000000 & 0.026869839851570 & 2.500000000000000 & 0.723130160148430 \\
\hline 0.150 & 1.999999694097679 & 1.992386888096835 & 0.007612806000845 & 1.000584210914877 & 0.999415483182802 \\
\hline 0.300 & 1.999999999999907 & 1.999668276101549 & $3.317238983577830 \mathrm{e}-04$ & 0.431255406963110 & 1.568744593036797 \\
\hline 0.450 & 2 & 2.000013827894327 & $1.382789432691212 \mathrm{e}-05$ & 0.011202289933945 & 1.988797710066055 \\
\hline 0.60 & 2 & 2.000037966559722 & $3.796655972232443 \mathrm{e}-05$ & 0.155941088089072 & 1.844058911910928 \\
\hline 0.750 & 2 & 2.000048484022029 & $4.848402202917157 \mathrm{e}-05$ & 1.488210270869652 & 0.511789729130348 \\
\hline 0.900 & 2 & 2.000058500354282 & $5.850035428212053 e-05$ & 4.755666607510483 & 2.755666607510483 \\
\hline 1.050 & 2 & 2.000068501034364 & $6.850103436395472 \mathrm{e}-05$ & 10.577561356069847 & 8.577561356069847 \\
\hline 1.200 & 2 & 2.000078501385237 & $7.850138523712857 \mathrm{e}-05$ & 18.946793694824862 & 16.946793694824862 \\
\hline 1.350 & 2 & 2.000088501782404 & $8.850178240438211 \mathrm{e}-05$ & 28.455464395393047 & 26.455464395393047 \\
\hline 1.500 & 2 & 2.000098502229882 & $9.850222988161406 \mathrm{e}-05$ & 35.323532419514116 & 33.323532419514116 \\
\hline 1.650 & 2 & 2.000108502727388 & $1.085027273881600 \mathrm{e}-04$ & 32.516687157414310 & 30.516687157414310 \\
\hline 1.800 & 2 & 2.000118503274898 & $1.185032748982628 \mathrm{e}-04$ & 9.546331217538949 & 7.546331217538949 \\
\hline 1.950 & 2 & 2.000128503872412 & $1.285038724123666 \mathrm{e}-04$ & 46.091414331622040 & 48.091414331622040 \\
\hline
\end{tabular}

Table (7) : Numerical results of IBDF2 ( $\left.\delta=1, \delta_{1}=0.000001\right)$ method and BDF2 method 


\section{Step size $=\mathbf{0 . 0 1 5}$ (Second case) \\ $\varepsilon y^{\prime \prime}+y^{\prime}=0, \varepsilon=0.01$}

\begin{tabular}{|c|c|c|c|c|c|}
\hline $\mathrm{X}$ & Theoretical solution & $\begin{array}{l}\text { Numerical solution of } \\
\text { IBDF2 method }\end{array}$ & Error of IBDF2 method & $\begin{array}{l}\text { Numerical solution of } \\
\text { BDF2 method }\end{array}$ & $\begin{array}{l}\text { Error of BDF2 } \\
\text { method }\end{array}$ \\
\hline 0.015 & 1.776869839851570 & 1.750000000000000 & 0.026869839851570 & 2.500000000000000 & 0.723130160148430 \\
\hline 0.150 & 1.999999694097679 & 1.992378390175124 & 0.007621303922556 & 1.000584210914877 & 0.999415483182802 \\
\hline 0.300 & 1.999999999999907 & 1.999649776111770 & $3.502238881363251 \mathrm{e}-04$ & 0.431255406963110 & 1.568744593036797 \\
\hline 0.450 & 2 & 1.999985327786715 & $1.467221328521617 \mathrm{e}-05$ & 0.011202289933945 & 1.988797710066055 \\
\hline 0.60 & 2 & 1.999999466268900 & $5.337311004272038 \mathrm{e}-07$ & 0.155941088089072 & 1.844058911910928 \\
\hline 0.750 & 2 & 1.999999983529931 & $1.647006886429381 \mathrm{e}-08$ & 1.488210270869652 & 0.511789729130348 \\
\hline 0.900 & 2 & 1.999999999614421 & $3.855786800954775 \mathrm{e}-10$ & 4.755666607510483 & 2.755666607510483 \\
\hline 1.050 & 2 & 1.999999999996986 & $3.014033467252375 \mathrm{e}-12$ & 10.577561356069847 & 8.577561356069847 \\
\hline 1.200 & 2 & 2.000000000000356 & $3.557154570899002 \mathrm{e}-13$ & 18.946793694824862 & 16.946793694824862 \\
\hline 1.350 & 2 & 2.000000000000020 & $2.042810365310288 \mathrm{e}-14$ & 28.455464395393047 & 26.455464395393047 \\
\hline 1.500 & 2 & 1.999999999999990 & $1.043609643147647 \mathrm{e}-14$ & 35.323532419514116 & 33.323532419514116 \\
\hline 1.650 & 2 & 1.999999999999987 & $1.310063169057685 \mathrm{e}-14$ & 32.516687157414310 & 30.516687157414310 \\
\hline 1.800 & 2 & 1.999999999999986 & $1.376676550535194 \mathrm{e}-14$ & 9.546331217538949 & 7.546331217538949 \\
\hline 1.950 & 2 & 1.999999999999986 & $1.376676550535194 \mathrm{e}-14$ & 46.091414331622040 & 48.091414331622040 \\
\hline
\end{tabular}

Table (8) : Numerical results of IBDF2 $(\delta=1)$ method and BDF2 method 


\section{Step size $=0.03($ First case $)$}

$\varepsilon y^{\prime \prime}+y^{\prime}=0, \varepsilon=0.02$

\begin{tabular}{|l|l|l|l|l|l|}
\hline $\mathrm{X}$ & Theoretical solution & $\begin{array}{l}\text { Numerical solution of } \\
\text { IBDF2 }(\mathrm{a}=\mathrm{b}=20) \text { method }\end{array}$ & $\begin{array}{l}\text { Error of IBDF2( } \mathrm{a}=\mathrm{b}=20) \\
\text { method }\end{array}$ & $\begin{array}{l}\text { Numerical solution of } \\
\text { BDF2( }(\mathrm{a}=\mathrm{b}=20) \text { method }\end{array}$ & $\begin{array}{l}\text { Error of BDF2(a=b=20) } \\
\text { method }\end{array}$ \\
\hline 0.03 & 1.776869839851570 & 1.937500000000000 & 0.160630160148430 & 2.50000000000000 & 0.723130160148430 \\
\hline 0.30 & 1.999999694097679 & 1.962370370282104 & 0.037629323815576 & 65.307824844192770 & 63.307825150095084 \\
\hline 0.60 & 1.999999999999907 & 1.977255316393915 & 0.022744683605992 & $6.442745538550658 \mathrm{e}+04$ & $6.442545538550658 \mathrm{e}+04$ \\
\hline 0.90 & 2 & 1.986293499429314 & 0.013706500570686 & $6.597142776784954 \mathrm{e}+07$ & $6.597142576784954 \mathrm{e}+07$ \\
\hline 1.20 & 2 & 1.991726905575670 & 0.008273094424330 & $6.755473984425363 \mathrm{e}+10$ & $6.755473984225363 \mathrm{e}+10$ \\
\hline 1.50 & 2 & 1.994974612986215 & 0.005025387013785 & $6.917605359838356 \mathrm{e}+13$ & $6.917605359838156 \mathrm{e}+13$ \\
\hline 1.80 & 2 & 1.996906458329869 & 0.003093541670131 & $7.083627888474251 \mathrm{e}+16$ & $7.083627888474251 \mathrm{e}+16$ \\
\hline 2.10 & 2 & 1.998048554068537 & 0.001951445931463 & $7.253634957797640 \mathrm{e}+19$ & $7.253634957797640 \mathrm{e}+19$ \\
\hline 2.40 & 2 & 1.998717299677139 & 0.001282700322861 & $7.427722196784783 \mathrm{e}+22$ & $7.427722196784783 \mathrm{e}+22$ \\
\hline 2.70 & 2 & 1.999102502483158 & $8.974975168420940 \mathrm{e}-04$ & $7.605987529507615 \mathrm{e}+25$ & $7.605987529507615 \mathrm{e}+25$ \\
\hline 3.00 & 2 & 1.999317903697922 & $6.820963020777171 \mathrm{e}-04$ & $7.788531230215801 \mathrm{e}+28$ & $7.788531230215801 \mathrm{e}+28$ \\
\hline 3.30 & 2 & 1.999431629746284 & $5.683702537164770 \mathrm{e}-04$ & $7.975455979740980 \mathrm{e}+31$ & $7.975455979740980 \mathrm{e}+31$ \\
\hline 3.60 & 2 & 1.999484477082621 & $5.155229173794407 \mathrm{e}-04$ & $8.166866923254760 \mathrm{e}+34$ & $8.166866923254760 \mathrm{e}+34$ \\
\hline 3.90 & 2 & 1.999500873785333 & $4.991262146674114 \mathrm{e}-04$ & $8.362871729412878 \mathrm{e}+37$ & $8.362871729412878 \mathrm{e}+37$ \\
\hline
\end{tabular}

Table (9) : Numerical results of IBDF2 ( $\left.\delta=1, \delta_{1}=0.0000001\right)$ method and BDF2 method . 


\section{Step size $=0.03$ (Second case) \\ $\varepsilon y^{\prime \prime}+y^{\prime}=0, \varepsilon=0.02$}

\begin{tabular}{|l|l|l|l|l|l|}
\hline $\mathrm{X}$ & Theoretical solution & $\begin{array}{l}\text { Numerical solution of } \\
\text { IBDF2(a=b=20) method }\end{array}$ & $\begin{array}{l}\text { Error of IBDF2(a=b=20) } \\
\text { method }\end{array}$ & $\begin{array}{l}\text { Numerical solution of } \\
\text { BDF2(a=b=20) method }\end{array}$ & $\begin{array}{l}\text { Error of BDF2(a=b=20) } \\
\text { method }\end{array}$ \\
\hline 0.03 & 1.776869839851570 & 1.967741935483871 & 0.190872095632301 & 2.500000000000000 & 0.723130160148430 \\
\hline 0.30 & 1.999999694097679 & 1.980266499505787 & 0.019733194591892 & 65.307824844192770 & 63.307825150095084 \\
\hline 0.60 & 1.999999999999907 & 1.988161078000563 & 0.011838921999344 & $6.442745538550658 \mathrm{e}+04$ & $6.442545538550658 \mathrm{e}+04$ \\
\hline 0.90 & 2 & 1.992899411326480 & 0.007100588673520 & $6.597142776784954 \mathrm{e}+07$ & $6.597142576784954 \mathrm{e}+07$ \\
\hline 1.20 & 2 & 1.995742358903052 & 0.004257641096948 & $6.755473984425363 \mathrm{e}+10$ & $6.755473984225363 \mathrm{e}+10$ \\
\hline 1.50 & 2 & 1.997447581668470 & 0.002552418331530 & $6.917605359838356 \mathrm{e}+13$ & $6.917605359838156 \mathrm{e}+13$ \\
\hline 1.80 & 2 & 1.998470124514628 & 0.001529875485372 & $7.083627888474251 \mathrm{e}+16$ & $7.083627888474251 \mathrm{e}+16$ \\
\hline 2.10 & 2 & 1.999083160954544 & $9.168390454559194 \mathrm{e}-04$ & $7.253634957797640 \mathrm{e}+19$ & $7.253634957797640 \mathrm{e}+19$ \\
\hline 2.40 & 2 & 1.999450620311540 & $5.493796884599167 \mathrm{e}-04$ & $7.427722196784783 \mathrm{e}+22$ & $7.427722196784783 \mathrm{e}+22$ \\
\hline 2.70 & 2 & 1.999670843172778 & $3.291568272223433 \mathrm{e}-04$ & $7.605987529507615 \mathrm{e}+25$ & $7.605987529507615 \mathrm{e}+25$ \\
\hline 3.00 & 2 & 1.999802807229115 & $1.971927708850352 \mathrm{e}-04$ & $7.788531230215801 \mathrm{e}+28$ & $7.788531230215801 \mathrm{e}+28$ \\
\hline 3.30 & 2 & 1.999881874673444 & $1.181253265556403 \mathrm{e}-04$ & $7.975455979740980 \mathrm{e}+31$ & $7.975455979740980 \mathrm{e}+31$ \\
\hline 3.60 & 2 & 1.999929243857753 & $7.075614224705262 \mathrm{e}-05$ & $8.166866923254760 \mathrm{e}+34$ & $8.166866923254760 \mathrm{e}+34$ \\
\hline 3.90 & 2 & 1.999957620210428 & $4.237978957233324 \mathrm{e}-05$ & $8.362871729412878 \mathrm{e}+37$ & $8.362871729412878 \mathrm{e}+37$ \\
\hline
\end{tabular}

Table (10) : Numerical results of IBDF2 $(\delta=0.55)$ method and BDF2 method . 